\title{
DEMOCRAZIA CON AGGETTIVI. L'INNOVAZIONE CONCETTUALE NELLA RICERCA COMPARATA
}

\section{di David Collier e Steven Levitsky}

La recente ondata universale di democratizzazioni ha posto gli studiosi di fronte alla sfida del confronto concettuale con una grande varietà di regimi postautoritari. Sebbene i nuovi regimi politici nazionali dell'America Latina, dell'Africa, dell'Asia e dell'ex mondo comunista condividano alcuni importanti attributi della democrazia, molti di essi presentano profonde differenze tra di loro e rispetto alle democrazie dei paesi industriali avanzati. E proprio per questo non pochi di essi non sono considerati democrazie a pieno titolo.

In questo articolo si sostiene che gli studiosi rispondono alla sfida perseguendo due obiettivi potenzialmente in contrasto. $\mathrm{Da}$ un lato, i ricercatori tentano di accrescere la differenziazione analitica per poter cogliere le diverse forme di democrazia che sono emerse. Dall'altro, si trovano di fronte al problema della validità dei concetti. Più specificamente: cercano di evitare il rischio dello stiramento dei concetti che sorge quando il concetto di democrazia viene applicato a casi per i quali, secondo gli standard accademici vigenti, esso non è appropriato ${ }^{1}$. Il risultato di questo comportamento è stato la proliferazione di forme

La versione inglese di questo saggio è apparsa su «World Politics», 49 (aprile 1997). Ringraziamo per $i$ validi suggerimenti Ruth Berins Collier, Larry Diamond, Andrew Gould, Peter Houtzager, Marcus Kurtz, Terry Karl, David Laitin, George Lakoff, Arend Lijphart, James Mahoney, Scott Mainwaring Carol Medlin, Gerardo Munck, Guillermo O'Donnell, Michael Pretes, Philippe Schmitter, Laura Stoker, Mark Turner, Samuel Valenzuela e $i$ partecipanti al Berkeley Working Group on Comparative Method. La partecipazione di Steven Levitsky a questa ricerca è stata agevolata da una National Science Foundation Graduate Fellowship, e il lavoro di David Collier a questo progetto presso il Center for Advanced Study in the Behavioral Sciences è stato sostenuto dal National Science Foundation Grant n. SBR-9022192.

1 Cfr. Sartori (1970) e Collier e Mahon (1993).

RIVISTA ITALIANA DI SCIENZA POLITICA / a. XXVII, n. 3, dicembre 1997 
concettuali alternative, incluso un sorprendente numero di sottotipi che comportano una democrazia «con aggettivi» ${ }^{2}$. Fra gli esempi che si possono trarre dalle centinaia di sottotipi comparsi, vi sono la «democrazia autoritaria», la «democrazia neopatrimoniale», la «democrazia dominata dai militari» e la «protodemocrazia».

Questa proliferazione si è verificata malgrado gli sforzi profusi da analisti di primo piano per standardizzare l'uso del termine democrazia sulla base delle definizioni procedurali offerte dalla tradizione inaugurata da Joseph Schumpeter e Robert A. $\mathrm{Dahl}^{3}$. Per alcuni importanti aspetti, questa standardizzazione ha avuto successo. Tuttavia, dal momento che la democratizzazione è continuata e l'attenzione si è concentrata su una serie di casi sempre più diversi, anche la proliferazione di sottotipi e di altre innovazioni concettuali è proseguita. Per cui, dato il rischio di una crescente confusione concettuale, il precedente sforzo di standardizzare l'uso deve essere adesso integrato dall'esame della struttura di pensiero che sottostà a queste diverse forme del concetto.

Questo articolo dà inizio a tale esame, concentrandosi sulle categorie qualitative ${ }^{4}$ impiegate nello studio di casi recenti di democratizzazione a livello di regimi politici nazionali, con particolare attenzione alle ricerche condotte sull'America Latina' Il nostro scopo è duplice: rendere più comprensibile la complessa struttura delle strategie alternative di innovazione concet-

2 L'espressione parallela «democrazia senza aggettivi» ha fatto la sua comparsa in America Latina nel corso di dibattiti fra osservatori alle prese con il problema della persistenza di forme di democrazia incomplete e condizionate. Cfr. ad esempio Krauze (1986).

3 Cfr. Schumpeter (1947); Dahl (1971).

4 Assieme alla categorie qualitative, che rappresentano il punto nodale di questa discussione, per poter comparare casi recenti di democratizzazione sono stati sviluppati indicatori quantitativi valutabili. In sede conclusiva, sarà producente raccogliere insieme le intuizioni offerte dalle strategie di innovazione concettuale impiegate in questi approcci alternativi. Tuttavia in questa fase a noi interessa compiere un passo prioritario essenziale: acquisire maggiori cognizioni sulle innovazioni concettuali introdotte dagli studiosi che usano categorie qualitative.

5 Non ci occupiamo pertanto in via prioritaria della letteratura sulle democrazie industriali avanzate, benché tale letteratura costituisca un importante punto di riferimento negli studi che stiamo esaminando. Solo in alcuni frangenti abbiamo preso in considerazione studi recenti di paesi che non fanno parte della fase del processo di democratizzazione attualmente in corso ma le cui democrazie, relativamente nuove, sono un elemento di comparazione negli studi che passeremo in rivista: ad esempio, la Colombia. Faremo inoltre alcuni riferimenti ad altri casi storici che sono stati utilizzati nella letteratura scientifica recente come importanti elementi di contrasto analitico. 
tuale che sono emerse ed esaminare vantaggi e svantaggi di tali strategie. Inizieremo con le ben note strategie di Sartori, consistenti nel salire o scendere lungo una scala di generalità - e volte, rispettivamente, a evitare lo stiramento concettuale e aumentare la differenziazione. Dal momento che questo approccio non può essere utilizzato per perseguire simultaneamente entrambi gli scopi, scopriremo che gli studiosi si sono spesso rivolti ad altre strategie: creare sottotipi «ridotti» di democrazia, «precisare» la definizione di democrazia aggiungendo attributi definitorii, sostituire il concetto sovrastante con il quale la democrazia è associata (ad esempio, da regime democratico a stato democratico).

Più in generale, questa analisi cerca di incoraggiare gli studiosi ad essere più attenti quando definiscono e usano dei concetti. I sottotipi e le altre forme concettuali esaminate in questa sede sono, in definitiva, componenti critiche delle principali argomentazioni sostantive presentate da questi ricercatori, e spesso anticipano la caratterizzazione complessiva del caso o dei casi in questione offerta dall'autore. Sono $\mathrm{i}$ «contenitori di dati» che danno l'idea dei fatti più significativi relativi ai regimi in discussione $e^{6}$. Se si vogliono descrivere $\mathrm{i}$ nuovi regimi adeguatamente, questi contenitori di dati devono essere utilizzati in modo chiaro e appropriato.

Una migliore descrizione, a sua volta, è essenziale per stabilire le cause e le conseguenze della democrazia, scopo che è cruciale in questo genere di letteratura. Molti studi, incluse opere famose di analisi storico-comparativa e sia vecchi che nuovi studi sui «requisiti sociali», hanno trattato la democrazia come un esito da spiegare 7 . Altre analisi hanno guardato all'impatto della democrazia e di specifici tipi di democrazia sulla crescita economica, sulla distribuzione del reddito, sulla liberalizzazione e sull'adattamento dell'economia e sui conflitti internazionali ${ }^{8}$. In questi studi, i risultati di accertamenti causali possono essere fortemente influenzati dal significato che l'autore attribuisce al

6 Sartori $(1970,1039)$.

7 Cfr. Moore (1966); Luebbert (1991); Rueschemeyer, E.H. Stephens e J.D. Stephens (1992); Lipset (1959); Lipset (1994); Londregan e Poole (1996); Przeworski e Limongi (1997).

8 Cfr. Przeworski e Limongi (1993); Bollen e Jackman (1985); Sirowy e Inkeles (1990); Remmer (1986); Stallings e Kaufman (1989); Russett (1993); Brown, Lynn-Jones e Miller (1996); Stepan e Skach (1993); Linz e Valenzuela (1994); O'Donnell (1994). 
termine democrazia9. Speriamo che questa discussione possa servire come un passo avanti verso una maggiore solidità e chiarezza di significato del concetto, che fornirà una base più adeguata per accertare relazioni causali.

$\grave{E}$ opportuno sottolineare che queste strategie di innovazione concettuale non sono assolutamente specifiche della ricerca qualitativa sui recenti processi di democratizzazione. Se ne può trovare traccia in molti ambiti concettuali, sia nelle scienze sociali che al di fuori di esse ${ }^{10}$. Un ulteriore obiettivo del presente articolo è perciò promuovere una più ampia comprensione del modo in cui i ricercatori qualitativi cercano di risolvere $\mathrm{i}$ fondamentali problemi della differenziazione analitica e della validità concettuale.

Le definizioni di democrazia nelle ricerche sui recenti processi di democratizzazione

Nella sua famosa analisi di «concetti messi in discussione», il filosofo W.B. Gallie sostiene che la democrazia è «il concetto politico par excellence soggetto a valutazioni critiche» ${ }^{11}$. Di conseguenza, ci si imbatte in dispute senza fine sul significato o sulla definizione appropriati che se ne possono dare. Tuttavia, scopo dell'analisi di Gallie non è semplicemente sottolineare l'importanza di tali dispute, bensì mostrare che una ricognizione dello status di un dato concetto come concetto discusso apre la possibilità di capire ogni significato all'interno del rispettivo contesto. Riferendosi alla democrazia, egli sostiene che «essendo la politica l'arte del possibile, gli obiettivi democratici saranno accresciuti o diminuiti a seconda del mutare delle circostanze», ed insiste che questi standard alternativi dovrebbero essere presi sul serio nei loro propri termini ${ }^{12}$.

In questo spirito, noi ci concentriamo sulle definizioni procedurali che sono state più ampiamente impiegate nella ricerca

9 Si vedano, ad esempio, Bollen e Jackman (1989, 613-616) e Russett (1993, 15-16).

$10 \mathrm{Per}$ un'analisi che ha per principale oggetto alcune di queste strategie, con riferimento ad un altro concetto delle scienze sociali, cfr. Collier (1995). Per la discussione condotta da linguisti e scienziati cognitivi sulla struttura intuitiva soggiacente a tali strategie, cfr. Cruse (1986, cap. 6); Lakoff (1987, capp. 2 e 6); Taylor (1989, capp. 2-3).

11 Gallie $(1956,184)$, in corsivo nell'originale.

12 Ibidem, 186; cfr. anche le pp. 178, 189, 190, 193. 
sui recenti processi di democratizzazione a livello di regimi politici nazionali. Queste definizioni si riferiscono a procedure democratiche, piuttosto che a politiche sostantive o ad altri esiti che potrebbero essere visti come democratici. Queste definizioni sono inoltre «minime», in quanto si concentrano deliberatamente sul più piccolo numero possibile di attributi che tuttora si reputa producano un sostegno sufficiente per la vita di una democrazia; ovviamente, non esiste un consenso generale sull'individuazione degli attributi necessari perché la definizione sia valida. Ad esempio, gran parte degli studiosi di cui ci occupiamo distinguono quelli che considerano i tratti più specificamente politici del regime dalle caratteristiche della società e dell'economia, basandosi sulla convinzione che queste ultime vadano più appropriatamente analizzate come potenziali cause o conseguenze della democrazia, piuttosto che come caratteri della democrazia in sé ${ }^{13}$.

All'interno di questo schema, ci concentriamo su una definizione «procedurale minima» che presuppone elezioni pienamente competitive con suffragio universale ed assenza di brogli massicci, combinate con effettive garanzie delle libertà civili, incluse la libertà di parola, riunione e associazione ${ }^{14}$. Tuttavia, non esiste assolutamente accordo fra gli studiosi su un'unica definizione. Alcuni, ad esempio, hanno creato una definizione «procedurale minima estesa» aggiungendo il criterio secondo cui i governi eletti devono avere l'effettivo potere di governare - criterio che, come vedremo in seguito, costituisce un problema cruciale in alcuni paesi.

\section{Le strategie di Sartori}

Consideriamo in primo luogo le strategie di Sartori per ottenere la differenziazione ed evitare lo stiramento concettuale. Sartori costruisce su un'intuizione basilare riguardo all'organiz-

13 Per una discussione delle definizioni procedurali, cfr. O'Donnell e Schmitter (1986, cap. 2); Huntington (1989); Schumpeter (1947) e Dahl (1971). Sulle definizioni minime, cfr. Di Palma $(1990,28)$ e Huntington $(1991,9)$. Sulla discussione delle caratteristiche della società e dell'economia come causa o conseguenza della democrazia, cfr. $\operatorname{Linz}(1975,182)$ e $\operatorname{Karl}(1990,2)$.

14 Cfr. O'Donnell e Schmitter (1986, 8; ma si veda anche il richiamo nella nota 33, infra); Diamond, Linz e Lipset (1989, XVI); Di Palma (1990, 16). Cfr. anche Linz $(1978,5)$. 
zazione dei concetti: un aspetto significativo del rapporto fra il significato dei concetti e il raggio dei casi a cui essi si applicano può essere inteso in termini di una «scala di generalità» ${ }^{15}$. Tale scala è basata su un modello di variazione inversa fra il numero degli attributi definitorii e il numero dei casi. Così, concetti con meno attributi definitorii in genere si applicano a più casi e si collocano perciò più in alto nella scala di generalità, mentre concetti con più attributi definitorii si applicano a meno casi e quindi stanno più in basso nella scala.

Differenziazione. Uno degli scopi di Sartori è mostrare come la differenziazione concettuale possa essere accresciuta scendendo la scala di generalità fino ad incontrare concetti che hanno molti attributi definitorii e si adattano ad una più ristretta gamma di casi. Tali concetti offrono le distinzioni a grana più fine, che per taluni disegni di ricerca si rivelano inservibili ${ }^{16}$. Questa discesa della scala viene spesso compiuta tramite la creazione di quelli che chiameremo i sottotipi «classici» di democrazia ${ }^{17}$. I sottotipi classici vengono considerati esempi pieni della definizione-radice ${ }^{18}$ di democrazia (root definition of democracy) in relazione alla quale vengono formati, nel momento stesso in cui essi vengono differenziati rispetto ad altri sottotipi classici di tale concetto. Così, «democrazia parlamentare», «democrazia multipartitica» e «democrazia federale» sono considerate tutte forme certamente democratiche (qualunque metro l'autore stia usando), e nel contempo ciascuna di esse è considerata un tipo particolare di democrazia (cfr. fig. 1). Nelle ricerche su casi recenti di democratizzazione, si può riscontrare ad esempio l'uso di sottotipi classici per ottenere differenziazione nell'importante

15 Sartori (1970) in realtà fa riferimento a una «scala di astrazione». Tuttavia, dal momento che il termine astratto è spesso inteso in contrasto con concreto, questa etichetta può creare confusione. Noi pertanto riteniamo che «scala di generalità» esprima più chiaramente il significato sottinteso.

16 Sartori $(1970,1041)$.

$17 \mathrm{Ci}$ riferiamo ad essi come sottotipi classici perché si accordano alla «classica» interpretazione della categorizzazione discussa da autori quali Lakoff (1987, 9 e passim) e Taylor (1989, cap. 2).

18 Facendo riferimento alla definizione-radice (root definition), non intendiamo sostenere che essa sia la definizione «corretta» del concetto che ci interessa (in questo caso, la democrazia). Si tratta semplicemente della definizione che, per un particolare autore, costituisce il punto di partenza nella formazione del sottotipo. Useremo occasionalmente l'espressione «concetto-radice» per riferirci al concetto (ancora una volta, nel presente contesto, la democrazia) che fa da punto di partenza per le varie innovazioni concettuali qui analizzate. 


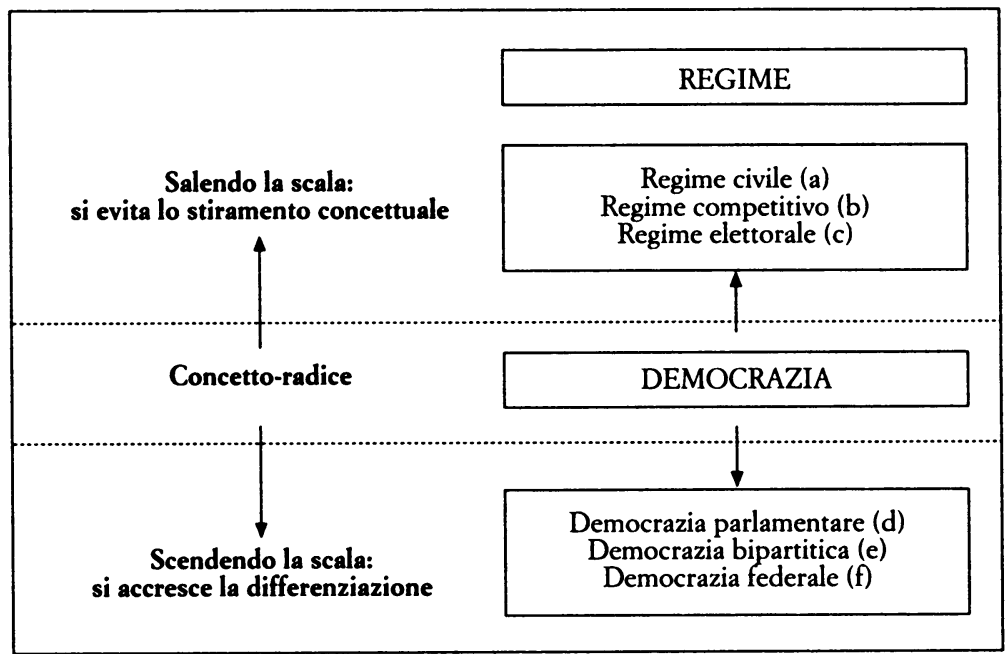
(a) Booth $(1989,26)$
(b) Collier e Collier $(1991,35)$
(d) $\operatorname{Linz}(1994,3)$
(c) Petras e Leiva $(1994,89)$
(e) Gasiorovski $(1990,113)$
(f) Gastil $(1990,35)$

FIG. 1. La scala di generalità: accrescere la differenziazione o evitare lo stiramento concettuale.

dibattito sulle conseguenze della democrazia parlamentare, contrapposta a quella presidenziale ${ }^{19}$.

Scendendo lungo la scala di generalità si ottiene un'utile differenziazione, e i sottotipi ora accennati svolgono un ruolo importante nella letteratura recente. I sottotipi formati in questa maniera possono però rendere vulnerabile l'analista allo stiramento concettuale, poiché presumono che i casi oggetto di discussione siano certamente delle democrazie. Se il caso particolare che viene studiato non è pienamente democratico, allora l'uso di questi sottotipi come strumento di differenziazione concettuale può non essere appropriato. Gli analisti vanno perciò alla ricerca di concetti che distinguano fra diversi gradi di democrazia, oltre a distinguere fra diversi tipi di democrazia. Dal momento che i sottotipi classici di democrazia contribuiscono soltanto al raggiungimento del secondo di questi obiettivi, essi non sono gli strumenti di differenziazione concettuale più co-

19 Cfr. Linz e Valenzuela (1994); Stepan e Skach (1993); Sartori (1994). 
munemente usati negli studi relativi i recenti processi di democratizzazione.

Evitare lo stiramento concettuale. La proposta di Sartori per evitare lo stiramento concettuale è quella di percorrere verso l'alto la scala di generalità per giungere a concetti che hanno un minor numero di attributi definitorii e corrispondentemente si adattano a una serie più ampia di casi ${ }^{20}$. Nel presente contesto, ciò chiama in causa concetti collocati al di sopra di quello di democrazia nella scala di generalità. Gli studiosi generalmente vedono la democrazia come un tipo specifico in relazione al sovrastante concetto di regime. Quindi, se hanno qualche dubbio sul fatto che un particolare caso sia realmente un regime democrati$c o$, possono salire nella scala e limitarsi a chiamarlo un regime.

Tuttavia, dato che spostarsi verso un concetto così generico quale è quello di regime comporta una grande perdita di differenziazione concettuale, gli studiosi si sono perlopiù mossi verso un livello intermedio (fig. 1), aggiungendo aggettivi al termine regime e dando in tal modo vita a sottotipi classici per differenziare specifici tipi di regime. I sottotipi risultanti rimangono più generici del concetto di democrazia, in quanto racchiudono non solo le democrazie ma anche alcune non-democrazie. Gli esempi includono un «regime civile», un «regime competitivo» e un «regime elettorale». In questo modo gli studiosi conseguono una qualche differenziazione concettuale in relazione al regime e allo stesso tempo non pregiudicano l'individuazione del caso in discussione come una democrazia. Un modo di procedere simile è adottato da quegli studiosi che usano un sinonimo al posto del termine regime, come accade quando scrivono di «governo civile»e «politica competitiva»"1.

Ascendere lungo la scala di generalità aiuta ad evitare lo stiramento concettuale, ma comporta un importante inconveniente. Dal momento che questi sottotipi rimangono più generici del concetto di democrazia, questo approccio conduce ad una perdita di differenziazione concettuale. Per cui, prese assieme, le due strategie di Sartori possono favorire l'uno o l'altro degli obiettivi, ma non entrambi contemporaneamente. Di conseguenza, molti studiosi si sono rivolti ad altre strategie.

20 Sartori $(1970,1041)$.

21 Cfr., rispettivamente, Wilson (1993) e Karl (1986). 


\section{Sottotipi ridotti}

Una strategia alternativa di innovazione concettuale, quella che consiste nel creare sottotipi «ridotti» («diminished»)22 può contribuire sia ad ottenere differenziazione, sia ad evitare lo stiramento dei concetti. $\grave{E}$ una strategia largamente usata nella letteratura sui processi recenti di democratizzazione. Per capire i sottotipi ridotti occorre sottolineare due punti cruciali. In primo luogo, contrariamente ai sottotipi classici discussi in precedenza, i sottotipi ridotti non sono esempi pieni della definizione-radice di «democrazia» utilizzata dall'autore che presenta il sottotipo. Ad esempio, «democrazia a suffragio limitato» e «democrazia tutelare» vengono intese come esempi incompleti di democrazia in quanto mancano di uno o più dei suoi attributi definitorii ${ }^{23}$. Di conseguenza, usando questi sottotipi l'analista si richiama ad una estensione più limitata della democratizzazione ed è perciò meno vulnerabile allo stiramento concettuale.

Il secondo punto riguarda la differenziazione. Dato che rappresentano una forma incompleta di democrazia, si potrebbe ritenere che i sottotipi ridotti abbiano meno attributi definitorii, con la conseguenza che si collocherebbero più in alto sulla scala di generalità e perciò fornirebbero una minore, e non una maggiore, differenziazione. Tuttavia, l'elemento distintivo dei sottotipi ridotti consiste nel fatto che essi in genere identificano specifici attributi della democrazia che sono mancanti, stabilendo così il carattere ridotto del sottotipo, nel momento stesso in cui identificano altri attributi della democrazia che sono invece presenti. Dal momento che specificano gli attributi mancanti, essi accrescono anche la differenziazione del concetto, e il sottotipo ridotto di fatto si riferisce ad una serie di casi diversa da quella chiamata in causa dalla definizione-radice di democrazia.

L'inclusione ed esclusione di casi che si verifica con un sottotipo ridotto, in contrapposizione all'ascesa o alla discesa della scala di generalità, può essere illustrata con gli esempi della

22 L'idea di sottotipi ridotti scaturisce dalla discussione di concetti radiali in Collier e Mahon (1993, 850-852). Cfr. anche Lakoff (1987, cap. 6).

$23 \mathrm{Dal}$ momento che sono esempi incompiuti, si potrebbe obiettare che non sono realmente «sottotipi» di democrazia. Ricorrendo a un termine della linguistica cognitiva, ci si può riferire ad essi come «etichette» concettuali che sono in parte derivate dal concetto di democrazia. Tuttavia, per evitare di doversi ripetutamente riferire a «sottotipi ed etichette», sembra più semplice, nella discussione che seguirà, chiamarli sottotipi. Cfr. Fauconnier e Turner (1994). 


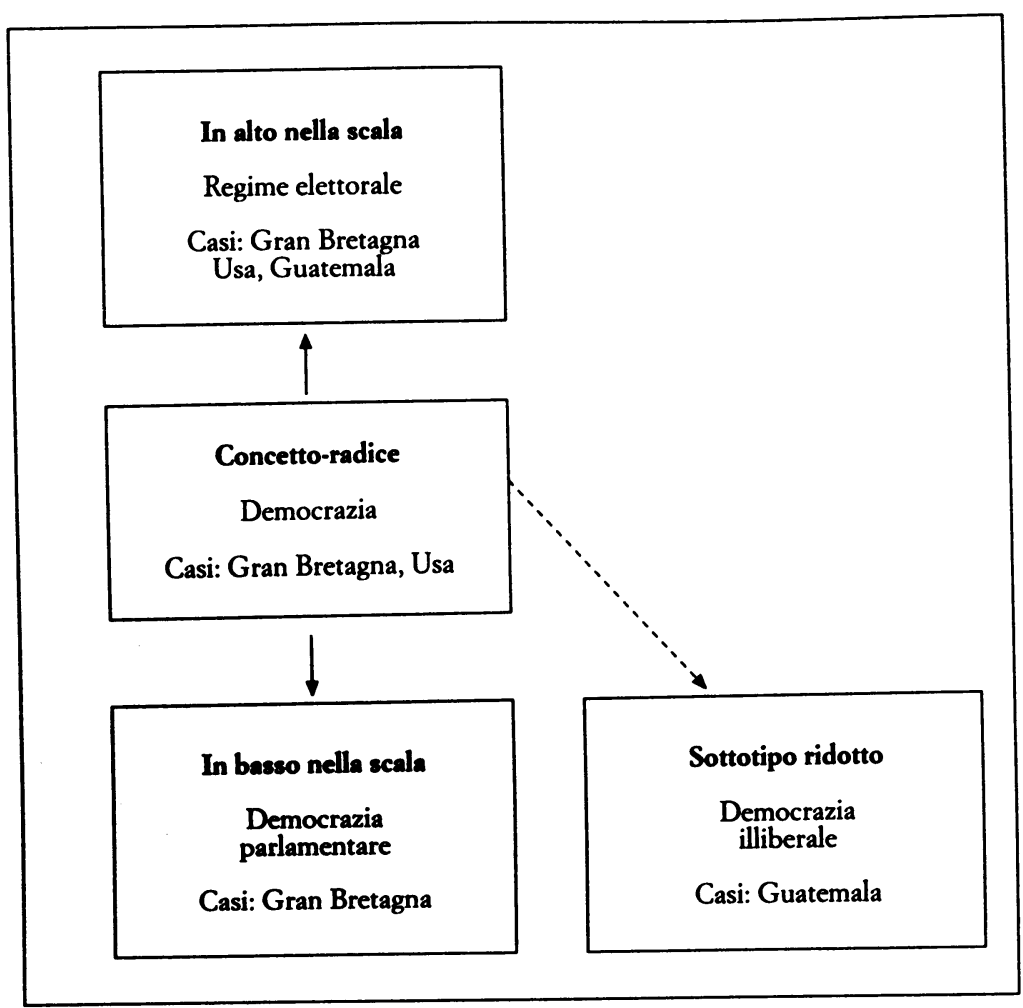

FIG. 2. Inclusione ed esclusione di casi: scala di generalità e sottotipi ridotti.

Gran Bretagna contemporanea, degli Stati Uniti e del Guatemala (fig. 2). La Gran Bretagna e gli Stati Uniti, ma probabilmente non il Guatemala (perlomeno sino alla metà degli anni novanta) potrebbero essere considerati democratici nei termini della definizione procedurale minima. Se ascendiamo nella scala di generalità, ci accorgiamo che il più ampio concetto di «regime elettorale» ${ }^{24}$ comprende tutti $\mathrm{e}$ tre $\mathrm{i}$ casi. Più in basso sulla scala, il classico sottotipo «democrazia parlamentare» includerebbe una delle due democrazie, cioè la Gran Bretagna. Di contro, il sottotipo ridotto «democrazia illiberale» includerebbe solo il Guafig. 1 .

$24 \mathrm{Si}$ intende che questo sottotipo ha il significato spiegato nella discussione della 


\section{Sottotipi ridotti a partire dalla definizione procedurale minima}

\begin{tabular}{|c|c|c|}
\hline (1a) & (1b) & (1c) \\
Attributo mancante: & Attributo mancante: & Attributo mancante: \\
Suffragio universale & Piena competitività & Libertà civili \\
Democrazia limitata (a) & Democrazia controllata (d) & Democrazia elettorale (g) \\
Democrazia maschile (b) & Democrazia monopartitica di fatto (e) & Democrazia dura (h) \\
Democrazia oligarchica (c) & Democrazia restrittiva (f) & Democrazia illiberale (i) \\
\hline
\end{tabular}

2. Sottotipi ridotti a partire dalla definizione procedurale minima estesa

Attributo mancante: Il governo eletto detiene l'effettivo potere di governare

Democrazia sorvegliata (j)

Democrazia protetta $(\mathrm{k})$

Democrazia tutelare (l)

(a) Archer $(1995,166)$

(b) Sorensen $(1993,20)$

(c) Hartlyn e Valenzuela $(1994,99)$

(d) Bagley $(1984,125)$

(e) Leftwich $(1993,613)$

(f) Waisman $(1989,69)$

(g) Hadenius $(1994,69)$

(h) O'Donnell e Schmitter $(1986,9)$. Questa è la nostra traduzione del loro termine democradura. In inglese i due au- tori parlano di limited democracy, lo stesso termine che abbiamo usato sopra in 1a, ma fanno chiaramente capire che il loro pensiero corrisponde a 1c.

(i) Emmerson $(1994,14)$

(j) Torres Riva $(1994,27)$. Questa è la nostra traduzione del suo democracia vigilada

(k) Loveman (1994, 108-11)

(l) Przeworski $(1988,60-61)$

FIG. 3. Democrazie parziali: esempi di sottotipi ridotti.

temala, il caso che specificamente non si adatta alla definizioneradice di democrazia ${ }^{25}$.

La figura 3 presenta alcuni esempi dei molti sottotipi ridotti che sono stati creati in relazione alle definizioni procedurale mi-

25 Riguardo alla democrazia illiberale, si veda la fig. 3. Circa i sottotipi ridotti, vanno sottolineati altri due punti. Primo: se gli studiosi non riescono ad identificare la definizione-radice di democrazia in relazione alla quale formano dei sottotipi, è difficile determinare se un dato sottotipo sia classico o ridotto. Secondo: il fatto che un sottotipo faccia riferimento a quella che potrebbe essere considerata una caratteristica «problematica» della democrazia non significa necessariamente che esso sia un sottotipo ridotto. Ad esempio, il concetto coniato da O'Donnell di «democrazia delegativa», che si riferisce a casi con una debole responsabilità orizzontale fra gli ambiti di governo, di fatto si accorda con la sua definizione minima di democrazia, dato che egli in tale definizione non include la responsabilità orizzontale. Cfr. O'Donnell $(1994,56)$. Pertanto, nell'uso che egli ne fa, la democrazia delegativa è un sottotipo classico. Per una discussione dei sottotipi che fa riferimento alle democrazie «problematiche», si veda una versione più estesa della presente analisi in Collier e Levitsky (1996, 20-26). La caratterizzazione sopra accennata della democrazia delegativa come sottotipo classico deve essere intesa come una correzione dell'accezione di tale sottotipo presentata in Collier (1995, 147-148). 
nima e procedurale minima estesa di democrazia menzionate in precedenza. In molti esempi, gli studiosi hanno creato sottotipi ridotti nei quali più di uno degli attributi costitutivi della democrazia sono mancanti, ma ai fini della nostra illustrazione ci concentriamo su esempi nei quali l'autore è stato ragionevolmente attento ad isolare un singolo attributo mancante. I sottotipi del primo gruppo (1a) si riferiscono a casi in cui l'attributo mancante è il suffragio universale. Qui troviamo termini come democrazia «maschile» o «oligarchica», che sono usati per sottolineare il contrasto fra casi contemporanei e casi storici precedenti all'avvento del suffragio universale. Dove l'attributo della piena competitività è mancante $(1 \mathrm{~b})$, come quando partiti importanti sono banditi dalla competizione elettorale, troviamo termini come democrazia «controllata» e «restrittiva». Dove le libertà civili sono incomplete $(1 \mathrm{c})$, gli studiosi hanno usato termini quali democrazia «elettorale» e «illiberale».

I sottotipi compresi nel gruppo finale (2), introdotti dagli studiosi che hanno creato la definizione procedurale minima estesa, forniscono un utile richiamo al fatto che il significato dei sottotipi dipende dalla definizione-radice di democrazia in relazione alla quale sono stati formati. Partendo da tale definizione, questi studiosi hanno introdotto sottotipi ridotti nei quali l'attributo mancante è l'effettivo potere del governo eletto di governare. Tali sottotipi pertanto non rispettano lo standard minimo procedurale esteso, sebbene rispettino lo standard procedurale minimo. Gli esempi che si riferiscono a casi ove le forze militari paiono detenere un eccessivo grado di potere politico includono la democrazia «protetta» e «tutelare».

I sottotipi ridotti, quindi, sono uno strumento utile per evitare lo stiramento dei concetti in casi che non possono dirsi completamente democratici. E forniscono inoltre un grado di differenziazione sufficiente a permettere la creazione di nuove categorie analitiche. Vari studiosi hanno sottolineato la necessità di procedere oltre una concettualizzazione dicotomica di autoritarismo e democrazia e di riconoscere il carattere «ibrido» o «misto» di molti regimi postautoritari ${ }^{26}$. La figura 3 suggerisce che questa ricognizione si è già verificata, e su una scala piuttosto ampia.

26 Cfr. Malloy (1987, 256-257); Conaghan e Espinal (1990, 555); Hartlyn (1994, 93-96); Karl (1995); Weffort (1992a, 89-90). 
Per paesi che non sono pienamente democratici sorge tuttavia un altro problema: se cioè non sarebbe meglio evitare di identificarli come sottotipi di democrazia; ad esempio, in casi di grosse violazioni delle libertà civili e/o di severe restrizioni alla competizione elettorale. Un esempio di questo interrogativo ci è offerto dalla decisione di Bruce Bagley di rifiutare i numerosi sottotipi ridotti di democrazia che sono stati applicati al periodo del Fronte Nazionale in Colombia (1958-74); fra questi egli include la democrazia «ristretta», «limitata», «oligarchica», «elitista» e «elitista-pluralista». Bagley caratterizza invece la Colombia come un sottotipo di autoritarismo: un «regime autoritario includente ${ }^{27}$. Altri studiosi hanno affrontato questo problema percorrendo verso l'alto la scala di generalità fino ad incontrare etichette come quelle di regime «civile», «competitivo» o «elettorale», che troviamo nella parte superiore della figura 1 . Una terza opzione è quella di utilizzare sottotipi che sottintendono un allontanamento dal concetto sul tipo di quelli sopra menzionati, come «democrazia di facciata», espressione nella quale l'aggettivo in sostanza cancella il carattere democratico del sottotipo. Gli studiosi dovrebbero essere consapevoli delle implicazioni analitiche e normative della scelta di formare sottotipi in relazione al concetto di democrazia o invece ad altri concetti.

\section{Precisare la definizione di democrazia}

Un'altra strategia di innovazione concettuale si incentra sulla definizione stessa di democrazia e si propone di «precisare» la definizione aggiungendo attributi definitoriii ${ }^{28}$. Quando il concetto viene esteso a nuovi àmbiti, i ricercatori possono trovarsi di fronte a un caso particolare che è classificato come una democrazia sulla base di una definizione comunemente accettata ma che però non è considerato pienamente democratico alla luce di una più ampia interpretazione condivisa del concetto. Questa imperfetta sovrapposizione fra il caso e la definizione formale può portare gli analisti a rendere espliciti uno o più criteri che sono implicitamente considerati parte del significato complessivo ma non sono inclusi nella definizione. Il risultato è

27 Bagley $(1984,125-127)$.

28 Cfr. Sartori $(1984,81)$ e Copi e Cohen $(1994,173-175)$. In Social Science Concepts (p. 42), Sartori usa anche la voce verbale «precisare» una definizione. 
una nuova definizione intesa a modificare il modo in cui un particolare caso è classificato. Benché possa essere vista come un innalzamento dei requisiti richiesti ad un regime per essere classificato come democratico, questo procedimento di precisazione della definizione può essere anche considerato un modo per adattare la definizione ad un nuovo contesto. Questa innovazione aumenta la differenziazione concettuale, aggiungendo un ulteriore criterio per stabilire la linea di divisione tra democrazia e non-democrazia. La strategia può quindi evitare anche lo stiramento dei concetti, giacché non applica l'etichetta «democrazia» a casi che, alla luce di questo nuovo criterio, sono visti dall'analista come non completamente democratici. Benché l'uso di questa strategia può scaturire dalla preoccupazione di adattare il concetto di democrazia per renderlo compatibile con un particolare contesto, la definizione modificata non dev'essere considerata rilevante unicamente per quel contesto. Essa può infatti fornire nuovi elementi di valutazione di altri casi rispetto ai quali l'importanza dei nuovi attributi definitorii non era stata, in precedenza, pienamente apprezzata.

Un esempio di precisazione della definizione ci è offerto dall'emergere di un criterio per identificare quel minimo procedurale esteso di cui abbiamo fatto cenno in precedenza. In parecchi paesi centroamericani, e in casi sudamericani come il Cile e il Paraguay, un lascito del governo autoritario è stato la persistenza di «ambiti riservati» al potere militare, sui quali i governi eletti hanno scarsa o nessuna autorità ${ }^{29}$. Pertanto, malgrado l'esistenza di elezioni libere o relativamente libere, i governi civili di tali paesi vengono considerati da taluni analisti privi di effettivo potere di governo. Alla luce di questi lasciti autoritari, e spesso in risposta alla pretesa secondo cui questi paesi, dal momento che tengono elezioni libere, sarebbero «democratici», alcuni studiosi hanno modificato la definizione procedurale minima di democrazia specificando il criterio esplicito per cui il governo eletto deve, in ragionevole misura, avere un effettivo potere di governare. Sulla base di questa definizione rivista, paesi come il Cile, El Salvador e il Paraguay sono stati esclusi da alcuni autori dall'insieme di casi classificati come democratici, nonostante abbiano tenuto elezioni relativamente libere ${ }^{30}$. Gli studiosi in que-

29 Cfr. Valenzuela $(1992,70)$. (1990)

30 Cfr. Karl (1990, 2); Valenzuela (1992) e Loveman (1994). Cfr. anche Rubin 
stione hanno quindi adattato la definizione per includervi esplicitamente un attributo che viene spesso dato per scontato negli studi sulle democrazie industriali avanzate ma è assente in questi casi latinoamericani.

Questa definizione rivista è stata sostanzialmente accettata, benché certamente non si sia manifestato un pieno accordo sul trattamento di casi specifici. Ad esempio, analizzando il Cile del periodo successivo al 1990, Rhoda Rabkin muove un'obiezione all'uso introdotto dagli studiosi che hanno adottato la definizione procedurale minima estesa, sostenendo che il problema del controllo civile sull'esercito non rappresenta una sfida al governo democraticamente eletto di portata tale da far qualificare il Cile come una democrazia «borderline»" ${ }^{31}$.

Altre due iniziative volte a precisare la definizione non hanno raccolto altrettanti consensi, ma possono essere utili per illustrare i problemi che sorgono adottando tale strategia. La prima di esse è rintracciabile in quella che potrebbe essere etichettata come una definizione tocquevilliana di democrazia, che sottolinea alcuni aspetti delle relazioni sociali. Analizzando il Brasile postautoritario, studiosi come Francisco Weffort e Guillermo O'Donnell sono stati colpiti dal fatto che i diritti di cittadinanza sono in larga misura minacciati dalle pervasive relazioni sociali semifeudali e autoritarie che continuano ad esistere in alcune regioni del paese. Alla luce di questa preoccupazione, essi hanno precisato la definizione di democrazia in modo tale da escluderne il Brasile. Così, Weffort aggiunge il requisito definitorio di «un qualche livello di eguaglianza sociale» perché un paese possa essere considerato democratico, e O'Donnell introduce una clausola simile ${ }^{32}$. Facendo proprio questo uso, i due autori ritengono di rimanere all'interno dello schema procedurale. $\mathrm{Ma}$ l'introduzione del problema delle relazioni sociali rappresenta un importante distacco dalle precedenti definizioni procedurali. Vedremo nella prossima sezione che O'Donnell in seguito è giunto ad un modo alternativo per incorporare questo insieme di preoccupazioni nella sua concettualizzazione della democrazia.

Un altro tentativo di precisare la definizione è scaturito dalla preoccupazione per il fatto che in molte nuove democrazie dell'America Latina e nei paesi ex comunisti i presidenti eletti a

31 Cfr. Rabkin (1992-93, 165).

32 Cfr. Weffort (1992b, 18); Weffort (1992a, 100-101); O'Donnell (1988, 297. 298); O'Donnell (1992, 48-49). 
volte fanno un uso estensivo del potere di decretazione, raggirano istituzioni democratiche come il parlamento e i partiti politici e governano con uno stile plebiscitario che pare celare in sé forti risvolti autoritari. Nel contesto latinoamericano, gli esempi di spicco includono Carlos Menem in Argentina, Fernando Collor de Mello in Brasile e, nel caso più estremo, Alberto Fujimori in Perù. La preoccupazione per queste tendenze autoritarie ha condotto taluni autori ad includere i controlli sul potere esecutivo fra i loro criteri procedurali di democrazia e di conseguenza ad escludere $\mathrm{i}$ casi di presidenzialismo non soggetto a limiti precisi ${ }^{33}$. Tuttavia, questa innovazione non è stata altrettanto ampiamente adottata.

Precisare la definizione può pertanto utilmente servire sia ad introdurre una più attenta differenziazione, sia ad evitare stiramenti concettuali, e i dibattiti collegati a questo tentativo hanno sollevato problemi essenziali circa il significato che gli studiosi intendono attribuire al termine «democrazia». Tuttavia la cautela è d'obbligo. Fra le strategie alternative di innovazione concettuale esaminate in questo articolo, la precisazione in un certo senso introduce il cambiamento più drastico: modifica la stessa definizione di democrazia. Se un'innovazione basata sulla precisazione viene ampiamente accettata, ha l'importante effetto di modificare il punto di partenza definitorio in riferimento al quale vengono perseguite tutte le altre strategie, sconvolgendo di fatto il «campo semantico» nel quale questi studiosi stanno lavorando ${ }^{34}$. Viceversa, l'introduzione di un nuovo sottotipo non influenza nello stesso modo il campo semantico. In una letteratura nella quale la confusione concettuale è un problema ricorrente, i guadagni analitici ricavabili dalla precisazione della definizione devono essere soppesati con il costo dello sconvolgimento del campo semantico.

Perciò è importante che gli studiosi evitino «il gerrymandering definitorio» ${ }^{35}$, nel senso di introdurre una nuova definizione ogni volta che incontrano un caso per qualche verso anomalo: Tuttavia, il contrasto tra il primo esempio (aggiungere il criterio

33 Fra gli autori che hanno adottato la responsabilità orizzontale nelle loro definizioni vi sono Schmitter e Karl $(1991,76,87)$ e Ball $(1994,45-46)$. O'Donnell e Schmitter $(1986,8)$ effettivamente includono questo requisito nella loro definizione formale, ma esso pare non svolgere alcun ruolo nella successiva analisi. 51-54).

34 Sul problema dello sconvolgimento del campo semantico, cfr. Sartori (1984,

35 Jennifer Whiting ci ha suggerito questo tema in una conversazione personale. 
dell'effettivo potere di governare) e il terzo (aggiungere la responsabilità orizzontale) mostra che gli studiosi possono di fatto imporre costruttivi limiti alle precisazioni. Nel primo esempio, l'incapacità dei governi eletti di esercitare effettivo potere era vista come un elemento invalidante del loro carattere democratico. Viceversa, nel terzo esempio, che coinvolge le rivendicazioni tiranniche di potere operate dal presidente, un dato cruciale è che questi presidenti sono leader eletti. Si può pertanto sostenere che è opportuno considerare che questi regimi presentino i requisiti minimi di democrazia ed evitare di avanzare precisazioni sintanto che: 1) essi mantengano elezioni presidenziali e un generale rispetto per le libertà civili; e 2) i partiti di opposizione non siano disciolti o messi fuorilegge (come è accaduto in Perù nel 1992).

In definitiva, l'attività di precisazione può far sorgere un problema, riportando all'interno della definizione di democrazia attributi che gli studiosi in precedenza avevano esplicitamente deciso di escludere. Un esempio è la preoccupazione per le relazioni sociali dell'approccio tocquevilliano. Può sembrare che gli autori che seguono questa strada rimangano all'interno di uno schema procedurale, in quanto sostengono che la partecipazione politica perde significato nel contesto di un'estrema ineguaglianza sociale. Tuttavia, questa innovazione concettuale reintroduce aspetti delle relazioni sociali in una maniera tale da rappresentare comunque uno spostamento notevole rispetto alle precedenti raccomandazioni circa gli attributi da includere nelle definizioni di democrazia.

\section{Spostare il concetto souraordinato}

Un'altra strategia di innovazione concettuale consiste nello spostamento del concetto sovraordinato in relazione al quale la democrazia viene vista come un esempio specifico, cioè come un sottotipo classico. Così, benché gli studiosi nella maggioranza dei casi vedano la democrazia come un sottotipo del concetto sovraordinato di «regime» (e i criteri procedurali per definire una democrazia discussi sopra potrebbero essere applicati, per via di semplice routine, al regime), una parte della letteratura recente ha inteso la democrazia come un sottotipo in relazione ad altri concetti sovraordinati, come appare da espressioni quali «governo democratico»e «stato democratico». Perciò, quando 


\begin{tabular}{|c|c|c|c|c|}
\hline & \multicolumn{2}{|c|}{$\begin{array}{l}\text { Abbassamento } \\
\text { del criterio di giudizio }\end{array}$} & \multirow{2}{*}{$\begin{array}{c}\begin{array}{c}\text { Punto } \\
\text { di partenza }\end{array} \\
\begin{array}{c}\text { Regime } \\
\text { democratico }\end{array} \\
\end{array}$} & \multirow{2}{*}{$\begin{array}{c}\begin{array}{c}\text { Innalzamento } \\
\text { del criterio } \\
\text { di giudizio }\end{array} \\
\text { Stato } \\
\text { democratico }\end{array}$} \\
\hline & $\begin{array}{c}\text { Situazione } \\
\text { democratica }\end{array}$ & $\begin{array}{c}\text { Governo } \\
\text { democratico }\end{array}$ & & \\
\hline 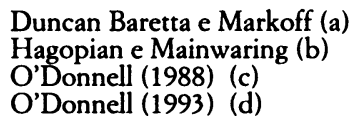 & Sì & $\begin{array}{l}\text { Sì } \\
\text { Sì }\end{array}$ & $\begin{array}{l}\text { No } \\
\text { No } \\
\text { No } \\
\text { Sì }\end{array}$ & No \\
\hline
\end{tabular}

(a) Duncan Baretta e Markoff $(1987,62)$

(c) O'Donnell $(1988,281)$

(b) Hagopian e Mainwaring $(1987,485)$

(d) O'Donnell $(1993,1360)$

FIG. 4. Spostamenti del concetto sovraordinato: la caratterizzazione del Brasile post1985.

a un determinato paese si applica l'etichetta «democratico», il significato dell'etichetta può variare a seconda del concetto sovraordinato al quale il termine viene connesso.

Una modifica del concetto sovraordinato può produrre un metro di misura alternativo per dichiarare che un particolare caso è una democrazia, senza peraltro modificare o slargare eccessivamente il concetto di «regime democratico». Come si può vedere nella figura 4 , tale strategia è stata impiegata dagli studiosi per creare un criterio di classificazione che può essere più o meno esigente. Ad esempio, ritenendo la democrazia brasiliana subito dopo il 1985 così poco istituzionalizzata da far apparire inappropriato l'uso dell'etichetta sovraordinata «regime», alcuni autori hanno parlato di una «situazione democratica». Questa distinzione segue l'esempio dell'analisi di Juan Linz del Brasile durante il primo periodo autoritario successivo al 1964: egli introdusse il concetto di «situazione autoritaria» per dar conto della debole istituzionalizzazione delle strutture politiche nazionali ${ }^{36}$. Altri autori, anch'essi alle prese con il Brasile post1985, hanno invece fatto riferimento ad un «governo democratico», onde suggerire che per quanto un particolare governo (cioè il Capo dello stato e la classe dirigente politica che sta immediatamente attorno al Capo dello stato) sia stato eletto democraticamente, il funzionamento corrente delle procedure de-

36 Cfr. Linz (1973). Malloy $(1987,236)$ usa l'espressione «momento democratico» per trasmettere un'opinione simile. 
mocratiche non era per questo necessariamente assicurato. Spostando il concetto sovraordinato dal regime al governo, gli studiosi abbassano lo standard richiesto per applicare l'etichetta «democratico».

In alternativa O'Donnell, spostando il concetto sovraordinato dal «regime» allo «stato», stabilisce un metro di giudizio più esigente per etichettare particolari paesi come democratici. Scrivendo dopo le elezioni presidenziali brasiliane del 1989, che hanno portato gli studiosi a reinterpretare il Brasile in chiave di regime democratico, O'Donnell pone degli interrogativi sul carattere democratico dello stato in Brasile, nonché in alcuni altri paesi sudamericani. Egli suggerisce che, nel contesto delle relazioni politiche «neofeudalizzate» e a volte «sultanistiche» esistenti in molte parti del paese, lo stato nazionale non tutela i diritti basilari di cittadinanza, e specificamente i diritti dei cittadini ad una corretta ed eguale protezione delle proprie relazioni sociali ed economiche. Questa carenza non può direttamente influenzare il funzionamento del regime, nel senso di danneggiare direttamente le elezioni e le libertà civili ad esse associate, che sono caratteri centrali dell'accordo procedurale di un regime democratico. Tuttavia, secondo O'Donnell, questa incapacità delle istituzioni legali e burocratiche del settore pubblico di proteggere e promuovere un più ampio insieme di diritti democratici dei cittadini è una caratteristica cruciale dello stato brasiliano. Per cui, benché riconosca che paesi come il Brasile hanno un «regime» democratico, egli li esclude dall'insieme dei paesi che ritiene abbiano «stati» democratici. Questo spostamento del concetto sovraordinato costituisce un altro modo per effettuare un accertamento più differenziato di cosa si debba intendere per caso incompleto di democrazia, stabilendo specificamente un criterio di giudizio più basso ed uno più elevato e dichiarando che certi paesi rispettano solo il criterio più basso ${ }^{37}$.

Nella prospettiva del mantenimento di una definizione procedurale di democrazia, questa innovazione può essere considerata una soluzione migliore al problema che O'Donnell ed altri hanno in un primo tempo cercato di affrontare formulando una definizione tocquevilliana. In questo modo, spostando il concetto sovrastante, il «regime» democratico conserva una definizione procedurale, e la preoccupazione per un più ampio funzio- 
namento della cittadinanza nel contesto di modelli autoritari di relazioni sociali è affrontata attraverso il concetto di stato.

In sintesi, la strategia consistente nell'effettuare spostamenti tra concetti sovraordinati alternativi può servire ad introdurre una più accurata differenziazione attraverso la creazione di una categoria analitica aggiuntiva. Quando è usata per abbassare il criterio di giudizio che consente di dichiarare l'appartenenza di un caso al genus democratico, la strategia può anche aiutare ad evitare lo stiramento del concetto di regime democratico. Quando è usata per innalzare il criterio di giudizio, non è rilevante rispetto al problema dello stiramento concettuale, perché non si preoccupa di evitare quello che potrebbe essere visto come l'errore di definire regime democratico un determinato caso. Offre invece informazioni aggiuntive sui casi che si ritiene abbiano regimi democratici.

\section{Osservazioni conclusive}

Abbiamo esaminato le strategie di innovazione concettuale usate dagli analisti dei recenti processi di democratizzazione quando cercano di sostenere una duplice sfida: accrescere la differenziazione analitica onde caratterizzare adeguatamente $i$ diversi regimi che sono emersi negli ultimi anni e mantenere la validità concettuale evitando lo stiramento dei concetti. Il nostro scopo è stato sia di rendere più comprensibile la complessa struttura di queste strategie, sia di valutarne i punti di forza e di debolezza. Anche quando hanno proceduto in modo intuitivo piuttosto che pienamente consapevole, questi studiosi hanno teso ad operare all'interno di tale struttura, che, come accennato sopra, non è assolutamente esclusiva della ricerca sui recenti processi di democratizzazione ${ }^{38}$. Ma nell'interesse della chiarezza concettuale e analitica è di gran lunga preferibile che si proceda consapevolmente, con una piena coscienza dei trade-offs esistenti fra le diverse strategie.

La figura 5 offre una panoramica di questa struttura analitica. L'innovazione concettuale si è verificata ai tre livelli del concetto-radice di democrazia, dei sottotipi e del concetto sovraordinato. Abbiamo osservato che le strategie suggerite da Sartori, 


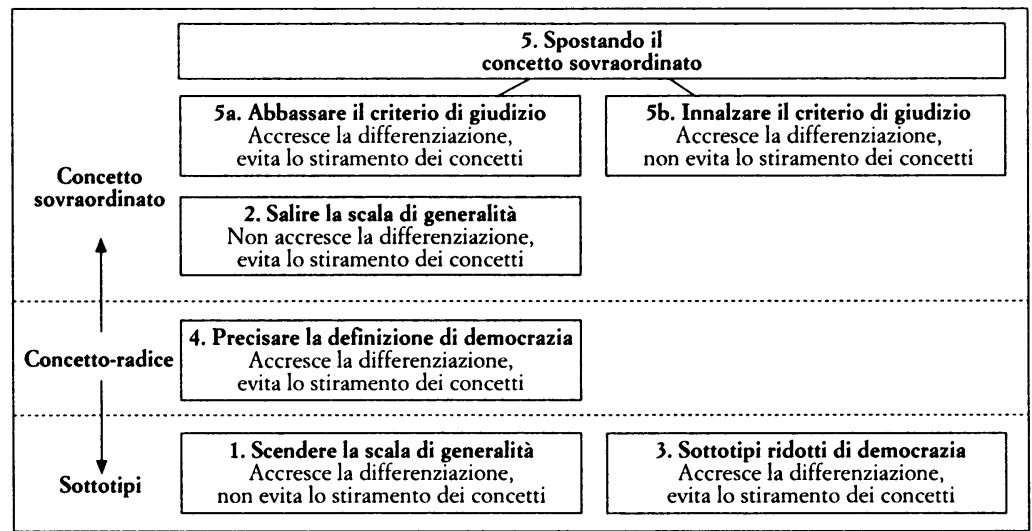

FIG. 5. Una valutazione delle innovazioni concettuali: i contributi per accrescere la differenziazione ed evitare lo stiramento dei concetti.

consistenti nello (1) scendere la scala di generalità sino a giungere ai sottotipi classici di democrazia e nel (2) salire la scala sino a giungere ai sottotipi classici di regime, possono servire utilmente o ad accrescere la differenziazione o ad evitare lo stiramento dei concetti, ma non possono fare entrambe le cose simultaneamente. Questi due obiettivi possono essere tuttavia raggiunti contemporaneamente (3) creando sottotipi ridotti, (4) precisando la definizione di democrazia tramite l'aggiunta di attributi definitorii, (5a) spostando i concetti sovraordinati, per abbassare il criterio di giudizio. Di contro (5b), spostare il concetto sovraordinato per innalzare i requisiti di una democrazia non serve ad evitare lo stiramento concettuale di fronte al concetto di regime democratico, ma introduce un ulteriore elemento di differenziazione.

Abbiamo inoltre sottolineato alcuni problemi che sono tipici di particolari strategie. I sottotipi ridotti sono utili per caratterizzare regimi ibridi, ma sollevano un problema: questi regimi devono di fatto essere trattati come sottotipi di democrazia oppure come sottotipi di autoritarismo o di un qualche altro concetto? La strategia che mira a precisare la definizione è soggetta al perenne problema delle dispute accademiche sulle definizioni di democrazia, nonché a quello di imporre un limite al gerrymandering definitorio. Per quanto non sia rilevante per il problema dello stiramento concettuale, la strategia dello sposta- 
mento del concetto sovraordinato allo scopo di innalzare il criterio di giudizio consente agli studiosi di introdurre nuovi quesiti analitici senza abbandonare una definizione procedurale di democrazia e regime.

In ultima analisi, queste strategie condividono due problemi. In primo luogo, data la complessità della loro struttura, il potenziale di confusione e difficoltà di comunicazione è considerevole. È indispensabile che gli studiosi definiscano e spieghino chiaramente la concezione di democrazia che stanno usando, onde collocarsi senza ambiguità in relazione a tale struttura.

In secondo luogo, questo genere di letteratura si trova a dover affrontare il fondamentale dilemma della proliferazione di concetti e termini, molti dei quali significano più o meno la stessa cosa. La conseguenza di questa situazione, ancora una volta, può essere una crescente confusione nella comunità scientifica. Sebbene vengano creati in parte perché gli studiosi stanno cercando di raggiungere una maggiore differenziazione e di evitare lo stiramento dei concetti, i nuovi termini possono anche essere introdotti con lo scopo di sviluppare etichette di particolare interesse, capaci di attrarre fortemente l'attenzione su nuove forme di democrazia ${ }^{39}$. Nella letteratura sui regimi politici nazionali prodotta negli ultimi trent'anni, sono state periodicamente introdotte importanti innovazioni analitiche in congiunzione con la creazione e/o sistematizzazione di concetti ed etichette concettuali che colgono in modo brillante importanti costellazioni di fenomeni: ad esempio «autoritarismo», «poliarchia», «autoritarismo burocratico», «corporativismo» e «democrazia consociativa ${ }^{40}$. Analogamente, l'invenzione di concetti ulteriori che svolgano lo stesso ruolo è un obiettivo importante nell'attuale studio dei regimi. Tuttavia, se la ricerca sulla democratizzazione degenera in una gara per vedere chi la spunta nel coniare il prossimo concetto famoso, lo studio comparativo dei regimi attraverserà serie difficoltà.

Perciò noi proponiamo un altro obiettivo fondamentale del-

$39 \mathrm{Per}$ tenere a mente quanto importanti possano essere etichette di richiamo, basta guardare all'impressionante evoluzione della teoria dei giochi, con la sua codificazione dei diversi modelli di interazione politica identificati da etichette quali «il dilemma del prigioniero», «il pollo», «la caccia al cervo», «la china scivolosa» e «la battaglia dei sessi». (1977).

40 Cfr. Linz (1964); Dahl (1971); O'Donnell (1973); Schmitter (1974); Lijphart 
l'uso dei concetti, che introduce un ulteriore bilanciamento rispetto agli scopi di raggiungere una maggiore differenziazione ed evitare lo stiramento concettuale. Oltre a perseguire questi scopi, gli studiosi dovrebbero mirare alla parsimonia ed evitare l'eccessiva proliferazione di nuovi termini e concetti. Altrimenti, i vantaggi derivanti dai raffinamenti concettuali discussi in questo articolo saranno sovrastati dalla confusione concettuale che ne deriverà.

[Traduzione di Marco Tarchi]

\section{Riferimenti bibliografici}

Archer, R.P. (1995), Party Strength and Weakness in Colombia's Besieged Democracy, in S. Mainwaring e T.R. Scully (a cura di), Building Democratic Institutions: Party Systems in Latin America, Stanford, Stanford University Press.

Bagley, B.M. (1984), Colombia: National Front and Economic Development, in R. Wesson (a cura di), Politics, Policies, and Economic Development in Latin America, Stanford, Hoover Institution Press.

Ball, A.R. (1994), Modern Politics and Government, Chatham, Chatham House (5. ed.).

Bollen, K.A. e R.W. Jackman (1985), Political Democracy and the Size Distribution of Income, in «American Sociological Review», vol. 50.

- (1989), Democracy, Stability, and Dichotomies, in «American Sociological Review», vol. 54.

Booth, J.A. (1989), Framework for Analysis, in J.A. Booth e M.A. Seligson (a cura di), Elections and Democracy in Central America, Chapel Hill, University of North Carolina Press.

Brown, M.E., S.M. Lynn-Jones e S.E. Miller (a cura di) (1996), Debating the Democratic Peace: An International Security Reader, Cambridge, MIT Press.

Collier, D. (1995), Trajectory of a Concept: 'Corporatism' in the Study of Latin American Politics, in P.H. Smith (a cura di), Latin America in Comparative Perspective: New Approaches to Method and Analysis, Boulder, Westview Press.

Collier, D. e S. Levitsky (1996), Democracy 'with Adjectives': Conceptual Innovation in Comparative Research, Working Paper n. 230, Notre Dame, The Kellogg Institute, University of Notre Dame.

Collier, D. e J.E. Mahon (1993), Conceptual 'Stretching' Revisited: Adapting Categories in Comparative Analysis, in «American Political Science Review», vol. 87. 
Collier, R.B. e D. Collier (1991), Shaping the Political Arena: Critical Junctures, the Labor Movement, and Regime Dynamics in Latin America, Princeton, Princeton University Press.

Conaghan, C.M. e R. Espinal (1990), Unlikely Transitions to Uncertain Regimes? Democracy without Compromise in the Dominican Republic and Ecuador, in «Journal of Latin American Studies», vol. 22.

Copi, I.M. e C. Cohen (1994), Introduction to Logic, New York, Macmillan (9. ed.).

Cruse, D.A. (1986), Lexical Semantics, Cambridge, Cambridge University Press.

Dahl, R.A. (1971), Polyarchy: Participation and Opposition, New Haven, Yale University Press; trad. it. Poliarchia. Partecipazione e opposizione, Milano, Angeli, 1980.

Diamond, L., J.J. Linz e S.M. Lipset (1989), Preface, in L. Diamond, J.J. Linz e S.M. Lipset (a cura di), Democracy in Developing Countries: Latin America, Boulder, Lynne Rienner.

Di Palma, G. (1990), To Craft Democracies: An Essay on Democratic Transitions, Berkeley, University of California Press.

Duncan Baretta, S. e J. Markoff (1987), Brazil's Abertura: Transition to What?, in Malloy e Seligson (1987).

Emmerson, D. (1994), Region and Recalcitrance: Questioning Democracy in Southeast Asia, paper presentato al congresso mondiale dell'International Political Science Association, Berlin.

Fauconnier, G. e M. Turner (1994), Conceptual Projection and Middle Spaces, Report n. 9401, Department of Cognitive Sciences, San Diego, University of California.

Gallie, W.B. (1956), Essentially Contested Concepts, in «Proceedings of the Aristotelian Society», vol. 56, London, Harrison and Sons.

Gasiorowski, M.J. (1990), The Political Regimes Project, in «Studies in Comparative International Development», vol. 25.

Gastil, R.D. (1990), The Comparative Survey of Freedom: Experiences and Suggestions, in «Studies in Comparative International Development», vol. 25.

Hadenius, A. (1994), The Duration of Democracy: Institutional vs. Socio-economic Factors, in D. Beetham (a cura di), Defining and Measuring Democracy, London, Sage.

Hagopian, F. e S. Mainwaring (1987), Democracy in Brazil: Problems and Prospects, in «World Policy Journal», vol. 4.

Hartlyn, J. (1994), Crisis-Ridden Elections (Again) in the Dominican Republic: Neopatrimonialism, Presidentialism, and Weak Electoral Oversight, in «Journal of Interamerican Studies and World Affairs», vol. 36.

Hartlyn, J. e A. Valenzuela (1994), Democracy in Latin America since 1930, in L. Bethell (a cura di), The Cambridge History of Latin America, Cambridge, Cambridge University Press, vol. 6.

Huntington, S.P. (1989), The Modest Meaning of Democracy, in R.A. 
Pastor (a cura di), Democracy in the Americas: Stopping the Pendulum, New York, Holmes \& Meier.

- (1991), The Third Wave: Democratization in the Late Twentieth Century, Norman, University of Oklahoma Press; trad. it. La terza ondata. I processi di democratizzazione alla fine del XX secolo, Bologna, Il Mulino, 1995.

Karl, T.L. (1986), Democracy by Design: The Christian Democratic Party in El Salvador, in G. Di Palma e L. Whitehead (a cura di), The Central American Impasse, London, Croom Helm.

- (1990), Dilemmas of Democratization in Latin America, in «Comparative Politics», vol. 23.

- (1995), The Hybrid Regimes of Central America, in «Journal of Democracy», vol. 6.

Krauze, E. (1986), Por una democracia sin adjetivos, Mexico City, Joaquín Mortiz/Planeta.

Lakoff, G. (1987), Women, Fire, and Dangerous Things: What Categories Reveal about the Mind, Chicago, University of Chicago Press.

Leftwich, A. (1993), Governance, Democracy, and Development in the Third World, in «Third World Quarterly», vol. 14.

Lijphart, A. (1977), Democracy in Plural Societies: A Comparative Exploration, New Haven, Yale University Press.

Linz, J.J. (1964), An Authoritarian Regime: Spain, in E. Allardt e Y. Littunen (a cura di), Cleavages, Ideologies and Party Systems: Contributions to Comparative Political Sociology, Transactions of the Westermarck Society, vol. 10, Helsinki, Academic Bookstore.

- (1973), The Future of an Authoritarian Situation or the Institutionalization of an Authoritarian Regime: The Case of Brazil, in A. Stepan (a cura di), Authoritarian Brazil: Origins, Policies, Future, New Haven, Yale University Press.

- (1975), Totalitarian and Authoritarian Regimes, in F.I. Greenstein e N.W. Polsby (a cura di), Handbook of Political Science, Reading, Addison-Wesley, vol. 3.

- (1978), The Breakdown of Democratic Regimes: Crisis, Breakdown and Reequilibration, Baltimore, The Johns Hopkins University Press; trad. it. parz. La caduta dei regimi democratici, Bologna, Il Mulino, 1981.

- (1994), Presidential or Parliamentary Democracy: Does It Make a Difference?, in Linz e Valenzuela.

Linz, J.J. e A. Valenzuela (a cura di) (1994), The Failure of Presidential Democracy, Baltimore, The Johns Hopkins University Press; trad. it. parz. Il fallimento del presidenzialismo, Bologna, Il Mulino, 1995.

Lipset, S.M. (1959), Some Social Requisites of Democracy: Economic Development and Political Legitimacy, in «American Political Science Review», vol. 53. 
- (1994), The Social Requisites of Democracy Revisited, in «American Sociological Review», vol. 59.

Londegran, J.B. e K.T. Poole (1996), Does High Income Promote Democracy?, in «World Politics», vol. 49.

Loveman, B. (1994), 'Protected Democracies' and Military Guardianship: Political Transitions in Latin America, 1979-1993, in «Journal of Interamerican Studies and World Affairs», vol. 36.

Luebbert, G.M. (1991), Liberalism, Fascism, or Social Democracy: Social Classes and the Political Origin of Regimes in Interwar Europe, New York, Oxford University Press.

Mainwaring S., G. O'Donnell e J.S. Valenzuela (a cura di) (1992), Issues in Democratic Consolidation: the New South American Democracies in Comparative Perspective, Notre Dame, University of Notre Dame Press.

Malloy, J.M. (1987), The Politics of Transition in Latin America, in Malloy e Seligson (1987).

Malloy, J.M. e M. Seligson (a cura di) (1987), Authoritarians and Democrats: Regime Transitions in Latin America, Pittsburgh, University of Pittsburgh Press.

Moore, B. Jr. (1966), Social Origins of Dictatorship and Democracy: Lord and Peasant in the Making of the Modern World, Boston, Beacon Press; trad. it. Le origini sociali della dittatura e della democrazia, Torino, Einaudi, 1969.

O'Donnell, G. (1973), Modernization and Bureaucratic-Authoritarianism: Studies in South American Politics, Institute of International Studies, Politics of Modernization Series n. 9, Berkeley, University of California.

- (1988), Challenges to Democratization in Brazil, in «World Policy Journal», vol. 5

- (1992), Transitions, Continuities, and Paradoxes, in Mainwaring, O’Donnell e Valenzuela (1992).

- (1993), On the State, Democratization, and Some Conceptual Problems: A Latin American View with Glances at Some Postcommunist Countries, in «World Development», vol. 21.

- (1994), Delegative Democracy, in «Journal of Democracy», vol. 5.

O'Donnell, G. e Ph. Schmitter (1986), Transitions from Authoritarian Rule: Tentative Conclusions about Uncertain Democracies, Baltimore, The Johns Hopkins University Press.

Petras J. e F.I. Leiva (1994), Democracy and Poverty in Chile: The Limits to Electoral Politics, Boulder, Westview Press.

Przeworski, A. (1988), Democracy as a Contingent Outcome of Conflicts, in J. Elster e R. Slagstad (a cura di), Constitutionalism and Democracy, Cambridge, Cambridge University Press.

Przeworski, A. e F. Limongi (1993), Political Regimes and Economic Growth, in «Journal of Economic Perspectives», vol. 7. 
- (1997), Modernization: Theories and Facts, in «World Politics», vol. 49.

Rabkin, R. (1992-93), The Alywin Government and 'Tutelary' Democracy: A Concept in Search of a Case?, in «Journal of Interamerican Studies and World Affairs», vol. 34

Remmer, K.L. (1986), The Politics of Economic Stabilization: IMF Standby Programs in Latin America, 1954-1984, in «Comparative Politics», vol. 19.

Rubin, H. (1990), One Step Away from Democracy, in «Journal of Democracy», vol. 1.

Rueschemeyer, D., E.H. Stephens e J.D. Stephens (1992), Capitalist Development and Democracy, Chicago, University of Chicago Press.

Russett, B. (1993), Grasping the Democratic Peace: Principles for a Post-Cold War World, Princeton, Princeton University Press.

Sartori, G. (1970), Concept Misformation in Comparative Politics, in «American Political Science Review», vol. 64.

- (1984), Guidelines for Concept Analysis, in G. Sartori (a cura di), Social Science Concepts: A Systematic Analysis, Beverly Hills, Sage.

- (1994), Comparative Constitutional Engineering: An Inquiry into Structures, Incentives, and Outcomes, New York, New York University Press; trad. it. Ingegneria costituzionale comparata, Bologna, Il Mulino, 1994.

Schmitter, Ph. (1974), Still the Century of Corporatism?, in «Review of Politics», vol. 36.

Schmitter, Ph. e T.L. Karl (1991), What Democracy Is... and Is Not, in «Journal of Democracy», vol. 2.

Schumpeter, J. (1947), Capitalism, Socialism and Democracy, New York, Harper; trad. it. Capitalismo, socialismo, democrazia, Milano, Etas Kompass, 1964.

Sirowy, L. e A. Inkeles (1990), The Effects of Democracy on Economic Growth and Inequality: A Review, in «Studies in Comparative International Development», vol. 25.

Sorensen, G. (1993), Democracy and Democratization: Process and Prospects in a Changing World, Boulder, Westview Press.

Stallings, B. e R. Kaufman (a cura di) (1989), Debt and Democracy in Latin America, Boulder, Westview Press.

Stepan, A. e C. Skach (1993), Constitutional Frameworks and Democratic Consolidation: Parliamentarism versus Presidentialism, in «World Politics», vol. 46.

Taylor, J.R. (1989), Linguistic Categorization: Prototypes in Linguistic Theory, Oxford, Oxford University Press (2. ed.).

Torres Rivas, E. (1994), La gobernabilidad centroamericana en los noventa, in «America Latina, Hoy», vol. 2.

Valenzuela, J.S. (1992), Democratic Consolidation in Post-Transitional 
Settings: Notion, Process, and Facilitating Conditions, in Mainwaring, O'Donnell e Valenzuela.

Waisman, C.H. (1989), Argentina: Autarkic Industrialization and Illegitimacy, in Diamond, Linz e Lipset.

Weffort, F. (1992a), Qual democracia?, São Paulo, Companhia das Letras.

- (1992b), New Democracies, Which Democracies?, Working Paper n. 198, Latin American Program, Washington, D.C., Woodrow Wilson International Center for Scholars.

Wilson, R. (1993), Continued Counterinsurgency: Civilian Rule in Guatemala, in Gills, Rocamora e Wilson (a cura di), Low Intensity Democracy: Political Power in the New World Order, London, Pluto Press. 